\title{
PERFIL EPIDEMIOLÓGICO DA ESQUISTOSSOMOSE EM COMUNIDADE PERIFÉRICA DO MUNICÍPIO DE JEQUIÉ-BA
}

\author{
Lucio Santos BORGES ${ }^{1}$ \\ Tuany Santos SOUZA ${ }^{2}$ \\ Roberto Lessa MOTTA ${ }^{3}$ \\ Bruno Del Sarto AZEVEDO ${ }^{4}$ \\ Joana Angélica Andrade DIAS ${ }^{5}$ \\ Ivône Gonçalves NERY ${ }^{5}$
}

\begin{abstract}
${ }^{1}$ Fisioterapeuta graduado pela Universidade Estadual do Sudoeste da Bahia.
${ }^{2}$ Graduanda de Farmácia da Universidade Estadual do Sudoeste da Bahia.

${ }^{3}$ Educador físico. Técnico da Vigilância Epidemiológica da Prefeitura Municipal Jequié-BA.

${ }^{4}$ Enfermeiro. Mestrando do Programa de Pós-graduação em Enfermagem e Saúde da Universidade Estadual do

Sudoeste da Bahia.

${ }^{5}$ Enfermeira. Mestre em Enfermagem. Professora do Departamento de Saúde II da Universidade Estadual do Sudoeste da Bahia.

Autor correspondente: brunodelsarto@outlook.com
\end{abstract}

Recebido em: 30/05/2013 - Aprovado em: 14/11/2014 - Disponibilizado em: 15/12/2014

RESUMO: A esquistossomose constitui-se ainda hoje como uma das parasitoses mais prevalentes do mundo e no Brasil. O município de Jequié, por sua vez, é uma das áreas endêmicas da doença, apresentando uma taxa de infecção aproximada de 1 infectado em cada 100 habitantes. Desta forma, o estudo objetiva analisar o perfil dos casos de esquistossomose em uma comunidade periférica do município de Jequié-BA, no ano de 2011, segundo características demográficas, de morbidade e de tratamento medicamentoso. Trata-se de um estudo descritivo, com abordagem quantitativa, que se baseia em dados secundários, oriundos do Programa de Controle da Esquistossomose (PCE), referentes à população da comunidade do Barro Preto, Jequié-BA, no ano de 2011. Entre o perfil de casos positivos para esquistossomose, observa-se uma maioria de indivíduos do sexo masculino, entre a faixa etária de 10 a 19 anos, com carga parasitária leve e tratados dois meses após a coleta do material fecal. Conclui-se que a falta de medidas socioeducativas, além da ausência e/ou insuficiência de saneamento básico, são determinantes para a manutenção dessa morbidade na população da comunidade em questão. Sendo assim, faz-se necessário o planejamento e execução de ações de educação em saúde, bem como a implementação de medidas sanitárias pela gestão pública municipal para o controle da esquistossomose.

Palavras-chave: Esquistossomose. Schistosoma mansoni. Parasitos. Saúde pública. Áreas de Pobreza.

\section{EPIDEMIOLOGICAL PROFILE OF SCHISTOSOMIASIS IN PERIPHERAL COMMUNITY OF THE CITY OF JEQUIÉ, BAHIA STATE, BRAZIL}

\begin{abstract}
Schistosomiasis constitutes one of the most prevalent parasitosis in the world and in Brazil. The city of Jequie is one of the endemic areas of this disease, with an approximate infection rate of one infected in every 100 inhabitants. Thus, the aim of this study was to analyze the profile of schistosomiasis cases of a peripheral community of Jequié, Bahia state, Brazil, according to demographic characteristics, morbidity and drug treatment. This is a descriptive study with a quantitative approach, which was based on secondary data derived from the Schistosomiasis Control Program (SCP), referring to the population of the community of Barro Preto, Jequié, in 2011. From a total of 311
\end{abstract}


positive cases of schistosomiasis, males between 10-19 years were majority. They presented light intensity infection and were treated two months after collection of fecal material. The lack of educational measures and/or inadequacy of sanitation are crucial factors to the maintenance of this morbidity in the analyzed community. Thus, the planning and execution of health education actions and the implementation of sanitary measures by municipal public management are necessary for schistosomiasis control.

Keywords: Schistosomiasis. Schistosoma mansoni. Parasites. Public health. Poverty Areas.

\section{INTRODUÇÃO}

A esquistossomose é uma das parasitoses mais prevalentes do mundo, constituindo-se ainda hoje como um dos principais problemas de saúde pública. Segundo a Organização Mundial de Saúde OMS (2012), aproximadamente 240 milhões de pessoas se encontram infectadas pela doença, sobretudo na África, Ásia e nas Américas.

Nas Américas, o Schistosoma mansoni é o único transmissor da doença e, apesar da diminuição da prevalência e morbidade da mesma ao longo do tempo, ainda existem cerca de 6 milhões de pessoas infectadas, a maioria delas vivendo na Venezuela e no nordeste brasileiro (WHO, 2009). No Brasil, A Bahia é o estado com a segunda maior área endêmica do país, no qual a esquistossomose está presente em mais de $60 \%$ de seus municípios. As localidades com as prevalências mais elevadas são encontradas em municípios das bacias dos rios Paraguaçu, Jequiriçá, Itapecuru, Contas e Jaguaribe (BRASIL, 2011).
Nesse sentido, o município de Jequié, localizado na região sudoeste da Bahia e cortado pelo rio de Contas, é uma das áreas endêmicas da doença, apresentando, no ano de 2008, um total de 1.506 casos de esquistossomose, o que representa, aproximadamente, 1 infectado em cada 100 habitantes (BRASIL, 2014).

Assim sendo, este estudo objetiva analisar o perfil dos casos de esquistossomose na comunidade do Barro Preto, Jequié-BA, no ano de 2011, segundo características demográficas, de morbidade e de tratamento medicamentoso.

\section{MÉTODO}

Trata-se de estudo descritivo, com abordagem quantitativa. Teve como campo o município de Jequié-BA e como cenário a comunidade do Barro Preto, localizada no bairro Joaquim Romão, cortado pelo Rio Jequiezinho. Esta comunidade foi escolhida como cenário devido ao elevado número de casos de esquistossomose. 
O estudo se baseia em dados secundários, oriundos do Programa de Controle da Esquistossomose - PCE, programa este que vem desempenhando diversas atividades, nelas compreendidas inquérito coproscópico, tratamento dos doentes, pesquisa malacológica e atividades de saneamento básico e educativas.

Para a obtenção dos dados, foram selecionados todos os casos de esquistossomose da comunidade do Barro Preto, registrados no Sistema de Informação do PCE (SISPCE), no ano de 2011, totalizando 311 (trezentos e onze) fichas. Utilizou-se como instrumento de coleta de dados um formulário elaborado pelos pesquisadores, o qual abrange dados relativos à caracterização demográfica do indivíduo, ao exame realizado (parasitológico de fezes) e ao tratamento medicamentoso utilizado.

Vale salientar que, no que diz respeito ao exame parasitológico de fezes, a presença de infecção é baseada no exame microscópico pelo método de Kato-Katz. Considera-se caso positivo para S. mansoni, ou outro parasita, se pelo menos um ovo for detectado na lâmina. O método de Kato-Katz permite a classificação da carga parasitária de acordo com o número de ovos por gramas de fezes (OPG) detectados. Sendo assim, a carga parasitária pode ser considerada leve (1-99), moderada (100-399) ou elevada $(\geq 400)$
(WHO, 2002). Deste modo, buscou-se coletar, por meio do formulário, as informações relativas à carga parasitária e à presença de outros parasitos na amostra de fezes.

Para a coleta de dados junto ao sistema de informação supracitado, os pesquisadores acessaram os dados por meio dos técnicos da Vigilância Epidemiológica da Secretaria Municipal de Saúde de Jequié, que atuavam, à época da realização do estudo, como preceptores do Programa de Educação pelo Trabalho para a Saúde/Vigilância em Saúde (PET Saúde/VS) da Universidade Estadual do Sudoeste da Bahia (UESB), Campus de Jequié.

Os dados coletados foram digitados no programa estatístico Epi Info, versão 3.5.1, armazenados, tratados e apresentados sob a forma de tabelas por meio dos programas Microsoft Excel e Microsoft Word e em seguida analisados.

Este artigo é um recorte da pesquisa intitulada "Vigilância em Saúde: um olhar sobre a dengue e esquistossomose em uma comunidade", aprovado no Comitê de Ética em Pesquisa da UESB, através do protocolo $n^{\circ}$ 108/2011, CAAE 0086.454.000-11.

\section{RESULTADOS}

Na comunidade do Barro Preto, de um total de 4.722 exames realizados, entre os 
meses de maio a julho de 2011, $311(6,6 \%)$ casos positivos de esquistossomose foram notificados no PCE. Nesta população, foram $232(74,6 \%)$ pessoas do gênero masculino e $73(23,5 \%)$ do feminino, tendo ainda $6(1,9 \%)$ notificações com este campo ignorado. Com base na distribuição por faixa etária dos indivíduos infectados (Tabela 1), verificou-se que a maior proporção destes $(32,8 \%)$ está entre a faixa de 10 a 19 anos.

Tabela 1 - Casos positivos de Esquistossomose por faixa etária, na comunidade do Barro Preto, Jequié-BA, no ano de 2011.

\begin{tabular}{l|l|l}
\hline Idade (anos) & $\mathbf{n}$ & \% \\
\hline 0 a 9 & 12 & $3,9 \%$ \\
$\mathbf{1 0}$ a 19 & 102 & $32,8 \%$ \\
$\mathbf{2 0}$ a 29 & 92 & $29,6 \%$ \\
$\mathbf{3 0}$ a 39 & 42 & $13,5 \%$ \\
$\mathbf{4 0}$ a 49 & 35 & $11,2 \%$ \\
$\mathbf{5 0}$ a 59 & 11 & $3,5 \%$ \\
60 ou mais & 17 & $5,5 \%$ \\
\hline Total & 311 & $100,0 \%$ \\
\hline
\end{tabular}

Fonte: Dados da pesquisa.

Das 311 notificações, 308 traziam consigo o dado relativo à carga parasitária do indivíduo, variando de 1 a 527 OPG. Observou-se uma alta prevalência de carga parasitária leve $(99,1 \%)$ entre os residentes do Barro Preto (Tabela 2).

Tabela 2 - Quantidade de ovos de Schistosoma mansoni por grama de fezes, na comunidade do Barro Preto, Jequié-BA no ano de 2011.

\begin{tabular}{l|l|l}
\hline OPG & $\mathbf{n}$ & \% \\
\hline 1 a 99 & 305 & $99,1 \%$ \\
100 a 399 & 2 & $0,6 \%$ \\
400 ou mais & 1 & $0,3 \%$ \\
\hline Total & 308 & $100,0 \%$ \\
\hline
\end{tabular}

Fonte: Dados da pesquisa.

Foram encontrados, ainda, outros parasitas (Ascaris lumbricoides, Taenia sp., Ancylostoma duodenale, dentre outros) nas fezes de $26(8,4 \%)$ sujeitos que se submeteram aos exames coproparasitológicos.

Outra variável observada na análise dos dados do PCE foi o intervalo entre a coleta do material fecal para a realização do exame parasitológico e o início do tratamento medicamentoso para os casos positivos (Tabela 3). A maioria dos casos positivos foram tratados num intervalo entre 2 meses $(56,2 \%)$.

Tabela 3 - Intervalo entre a coleta do material fecal e o tratamento medicamentoso para Esquistossomose, na comunidade do Barro Preto, Jequié-BA, no ano de 2011.

\begin{tabular}{l|l|l}
\hline Intervalo & $\mathbf{n}$ & $\mathbf{\%}$ \\
\hline $\mathbf{1}$ mês & 10 & $3,2 \%$ \\
$\mathbf{2}$ meses & 173 & $56,2 \%$ \\
$\mathbf{3}$ meses & 124 & $40,3 \%$ \\
$\mathbf{4}$ meses & 1 & $0,3 \%$ \\
\hline Total & 308 & $100,0 \%$ \\
Fonte: Dados da pesquisa.
\end{tabular}


No que se refere ao efetivo quantitativo de casos tratados e não tratados, $25(8 \%)$ casos positivos para esquistossomose não receberam o medicamento, sendo este $o$ praziquantel.

\section{DISCUSSÃO}

A maior predisposição do sexo masculino para a esquistossomose se justifica por variáveis culturais e comportamentais. A observação social demonstra que os indivíduos deste gênero são mais expostos ao ambiente peridomiciliar durante as atividades de lazer, como banho, pesca e práticas esportivas (GUIMARAES; TAVARESNETO, 2006; PALMEIRA et al., 2010).

Com base nos achados relativos à faixa etária, verificou-se que a maior parte dos indivíduos parasitados $(32,8 \%)$ tem entre $10 \mathrm{e}$ 19 anos de idade, o que é corroborado por outros estudos que também mostram uma maior prevalência de infecção entre adolescentes e adultos jovens (BINA; PRATA, 2003; KABATEREINE et al., 2004; PALMEIRA et al., 2010). Tal predisposição também pode ser explicada pelo contato mais assíduo dos jovens com as águas em atividades recreativas e de lazer (CARDIM et al., 2011).

Observou-se uma alta prevalência de carga parasitária leve $(99,1 \%)$ entre os residentes da comunidade do Barro Preto. Entretanto, embora a maior intensidade da infecção seja um fator importante, ele não é o único responsável pela determinação das formas graves, especialmente a hepatoesplênica (BINA; PRATA, 2003). Esta, por vezes, depende de fatores genéticos ou da reação mais intensa do organismo do indivíduo à doença (PRATA, 2010).

Logo, tal dado não deve diminuir a intensidade do planejamento e execução das medidas de controle da esquistossomose, especialmente aquelas voltadas ao saneamento básico e à educação em saúde, já que ausência de ambos mostra-se como importantes determinantes para a transmissão e estabelecimento dessa parasitose (ROLLEMBERG et al., 2011; CANTANHEDE; FERREIRA; MATTOS, 2011; WHO, 2012, 2013; RIBEIRO et al., 2013).

$\begin{array}{lrrr}\text { Salienta-se } & \text { que } & \text { as } & \text { técnicas } \\ \text { parasitológicas de } & \text { fezes } & \text { variam }\end{array}$
consideravelmente quanto à sensibilidade, dependendo da quantidade de fezes examinadas, do número de ovos excretados e de fatores inerentes à perda intrínseca durante a realização do procedimento (GARGIONI et al., 2008).

O método utilizado (Kato-Katz), por sua vez, é o preconizado pela OMS devido às facilidades operacionais e baixo custo. 
Contudo, estudos como o de Siqueira et al. (2011) e de Enk et al. (2008) têm evidenciado que o exame da amostra de material fecal em uma única lâmina (metodologia adotada pelo PCE no Brasil) subestima significativamente a prevalência da esquistossomose. Nesse sentido, a sensibilidade do método aumenta sobremaneira quando se eleva o número de amostras diferentes de material fecal e de lâminas examinadas, podendo alcançar um quantitativo quase quatro vezes maior de casos positivos identificados. Por essa razão, há a possibilidade de grande subnotificação de casos no Barro Preto devido a um número considerável de falsos negativos.

As infecções por dois ou mais parasitos intestinais estão frequentemente associadas, contudo, poucos estudos informam a esse respeito, o que dificulta a interpretação e comparação dos resultados (BROOKER et al., 2006). Sendo assim, a relação entre $S$. mansoni e outros parasitos intestinais necessita de maior investigação.

O procedimento adotado pela Secretaria Municipal de Saúde de Jequié nos casos em que são encontrados outros parasitas nas fezes dos indivíduos, além do Schistosoma, é a disponibilização dos resultados dos exames aos infectados, para que os mesmos possam, assim, procurar assistência e providenciar seu respectivo tratamento.
Quanto ao intervalo entre a coleta do material fecal para a realização do exame parasitológico e o início do tratamento medicamentoso para os casos positivos, a maioria dos casos positivos foram tratados num intervalo entre 2 e 3 meses. Neste caso, quanto mais breve o intervalo entre a coleta e o tratamento, diminuem-se os riscos de complicações para o paciente, sendo que o prolongamento deste intervalo pode ser resultado de vários fatores, tais como logística inadequada, alta demanda de exames para um quantitativo reduzido de profissionais, deficiência de espaço e estrutura do centro de endemias local.

No que concerne ao número de indivíduos efetivamente tratados, 92\% dos casos receberam o praziquantel, em dose única, droga que apresenta resultados positivos na eliminação do parasita (CARDIM et al., 2011). O não tratamento de $25(8 \%)$ pessoas pode ter sido decorrente de contraindicação ao medicamento ou ainda por recusa ou ausência das mesmas no momento do retorno do agente de combate às endemias para a disponibilização do praziquantel. Este número, apesar de reduzido, deve ser visto como possível fator contribuinte para a persistência da esquistossomose na comunidade e no município. Por esta razão, ratifica-se a importância da implementação de ações de educação em saúde, sobretudo 
aliadas a estratégias de coparticipação, reforçando não só a profilaxia, mas também a necessidade do tratamento para a redução da prevalência da doença (CARDIM et al., 2011; RIBEIRO et al., 2013).

\section{CONCLUSÃO}

Os resultados obtidos no estudo revelam, para a comunidade periférica do Barro Preto, uma maior proporção de casos positivos de esquistossomose entre indivíduos do sexo masculino, jovens, com carga parasitária leve e tratados dois meses após o material fecal ter sido coletado.

A falta de medidas socioeducativas, a pouca ou má informação sobre as características da parasitose quanto aos meios de contaminação e às formas de tratamento, além da ausência e/ou insuficiência de saneamento básico são determinantes para a manutenção dessa morbidade na população exposta da comunidade em questão.

Dessa forma, faz-se necessário o planejamento e execução de ações de educação em saúde, bem como a implementação de medidas sanitárias pela gestão pública municipal para o controle da esquistossomose em regiões periféricas do município de Jequié, a exemplo da comunidade do Barro Preto.
Assim, torna-se fundamental a integração entre os órgãos públicos, o setor saúde e a comunidade, priorizando a esquistossomose como um problema que ultrapassa as questões puramente biológicas do processo saúde-doença, uma vez que tal agravo é determinado e condicionado por um conjunto de fatores sociais, culturais e econômicos, devendo, portanto, seu controle e erradicação ser encarados como responsabilidade de todos.

\section{REFERÊNCIAS}

BINA, J. C.; PRATA, A. Esquistossomose na área hiperendêmica de Taquarendi. I Infecção pelo Schistosoma mansoni e formas graves. Rev. Soc. Bras. Med. Trop., Uberaba, v. 36, n. 2, p. 211-6, 2003.

BRASIL. DATASUS - Departamento de Informática do SUS. PCE - Programa de Controle da Esquistossomose. 2014.

Disponível em:

$<$ http://tabnet.datasus.gov.br/cgi/tabcgi.exe?si nan/pce/cnv/pce.def>. Acesso em: 04 maio 2014.

BRASIL. Ministério da Saúde. Secretaria de Vigilância em Saúde. Sistema Nacional de Vigilância em Saúde: relatório de situação: Bahia. 5. ed. Brasília: Ministério da Saúde, 
2011. (Série C. Projetos, Programas e Relatórios)

BROOKER, S. et al. Contrasting patterns in the small-scale heterogeneity of human helminth infections in urban and rural environments in Brazil. Int. J. Parasitol., v. 36, p. 1143-51, 2006.

CARDIM, L. L. et al. Análises espaciais na identificação das áreas de risco para a esquistossomose mansônica no Município de Lauro de Freitas, Bahia, Brasil. Cad. Saúde Pública, Rio de Janeiro, v. 27, n. 5, p. 899908, 2011.

ENK, M. J. et al. The effect of the number of stool samples on the observed prevalence and the infection intensity with Schistosoma mansoni among a population in an area of low transmission. Acta Trop., Basel, v. 108, n. 23, p. 222-8, nov.-dec. 2008.

GARGIONI, C. et al. Utilização de método sorológico como ferramenta diagnóstica para implementação da vigilância e controle da esquistossomose no Município de Holambra, São Paulo, Brasil. Cad. Saúde Pública, Rio de Janeiro, v. 24, n. 2, p. 373-9, 2008.

GUIMARAES, I. C. S.; TAVARES-NETO, J. Transmissão urbana de esquistossomose em crianças de um bairro de Salvador, Bahia.

Rev. Soc. Bras. Med. Trop., Uberaba, v. 39, n. 5, p. 451-5, 2006.

KABATEREINE, N. B. et al. Epidemiology and geography of Schistosoma mansoni in Uganda: implications for planning control.

Trop. Med. Int. Health, Oxford, v. 9, n. 3, p. 372-80, 2004.

PALMEIRA, D. C. C. et al. Prevalência da infecção pelo Schistosoma mansoni em dois municípios do Estado de Alagoas. Rev. Soc. Bras. Med. Trop., Uberaba, v. 43, n. 3, p. 313-7, 2010.

PRATA, A. Esquistossomose Mansônica. In: VERONESI, R.; FOCACCIA, R. Tratado de Infectologia. Volume 2. 4 ed. São Paulo: Atheneu, 2010. p. 1859-82.

RIBEIRO, D. F. et al. Educação em saúde: uma ferramenta para a prevenção e controle de parasitoses. Revista da Universidade Vale do Rio Verde, Três Corações, v. 11, n. 2, p. 300-10, 2013.

ROLLEMBERG, C. V. V. et al. Aspectos epidemiológicos e distribuição geográfica da esquistossomose e geo-helmintos, no Estado de Sergipe, de acordo com os dados do Programa de Controle da Esquistossomose. 
Rev. Soc. Bras. Med. Trop., Uberaba, v. 44, n. 1, p. 91-6, 2011.

SIQUEIRA, L. M. V. et al. Evaluation of two coproscopic techniques for the diagnosis of schistosomiasis in a low-transmission area in the state of Minas Gerais, Brazil. Mem. Inst. Oswaldo Cruz, Rio de Janeiro, v. 106, n. 7, p. 844-50, nov. 2011.

\section{WORLD HEALTH ORGANIZATION} (WHO). Elimination of schistosomiasis in low transmission areas: Salvador, Bahia, 1819 Aug. 2008. Geneva: WHO, 2009. Report of the WHO informal consultation.

WORLD HEALTH ORGANIZATION

(WHO). Prevention and control of schistosomiasis and the soil-transmitted helminthiasis: Report of a WHO Expert Committee. Geneva: WHO, 2002.

\section{WORLD HEALTH ORGANIZATION} (WHO). Schistosomiasis: progress report 2001-2011 and strategic plan 2012-2020. Geneva: WHO, 2012.

\section{WORLD HEALTH ORGANIZATION}

(WHO). Schistosomiasis: number of people treated in 2011. Weekly epidemiological record, Geneva, v. 88, n. 8, p 81-8, 2013. 